\title{
Viabilidad de semillas de guayacán (Guaiacum sanctum L., Zygophyllaceae) posterior a dos tratamientos pregerminativos
}

\author{
Seed viability of lignum vitae (Guaiacum sanctum L., Zygophyllaceae) after two pregerminative \\ treatments \\ DOI 10.5377pc.v0i16.8093
}

\author{
Allison Flores Barahona ${ }^{1}$ \\ Lilian Ferrufino Acosta ${ }^{2}$ \\ Virna López Castro ${ }^{3}$
}

\section{RESUMEN}

Guaiacum sanctum $L$. es una especie arbórea de crecimiento lento que pertenece a la familia Zygophyllaceae, habita en el bosque seco subtropical y tropical. El objetivo de este estudio fue analizar la viabilidad de semillas de guayacán, posterior a dos tratamientos pregerminativos. La colecta de semillas se realizó en las cercanías del estadio Carlos Miranda, en el departamento de Comayagua, Honduras. Una vez colectadas las semillas se empleó corte longitudinal en la testa y lixiviación, como tratamientos pregerminativos y posteriormente se aplicó pruebas de flotabilidad y de tetrazolio para el análisis de viabilidad. Se obtuvo como resultados un mayor número de semillas viables, registrando los porcentajes de viabilidad más altos, en aquellas a las que no se les aplicó tratamiento pregerminativo. En cuanto a las pruebas de viabilidad se demostró que la más efectiva es la de tetrazolio, ya que permite observar de forma directa el embrión y el estado de los tejidos embrionarios. El análisis de viabilidad contribuyó a conocer la posible influencia de dichos tratamientos en la viabilidad de las semillas. Estos son factores importantes ya que se encuentran relacionados con la germinación de esta especie vegetal de especial interés económico y forestal.

Palabras clave: Lixiviación, porcentaje de viabilidad, prueba de tetrazolio, corte testa, Honduras.

\footnotetext{
${ }^{1}$ Autora, estudiante de Biología. Facultad de Ciencias, UNAH. allison.flores@unah.hn.

${ }^{2}$ Asesora. Profesora de la Escuela de Biología, Facultad de Ciencias, UNAH: lilian.ferrufino@ unah.edu.hn

${ }^{3}$ Asesora. Profesora de la Escuela de Biología, Facultad de Ciencias, UNAH: virna.lopez@ unah.edu.hn
} 


\section{ABSTRACT}

Guaiacum sanctum L. is a slow-growing tree species that belongs to the family Zygophyllaceae, inhabits the subtropical and tropical dry forest. The objective of this study was to analyze the viability of lignum vitae seeds, after two pregerminative treatments. The seed were collected nearly Carlos Miranda Stadium in Comayagua department, Honduras. Once the seeds were collected, seed coat incision and lixiviation were applied, as pregerminative treatments and subsequently applied buoyancy and tetrazolium tests for the viability analysis. A higher number of viable seeds was obtained, registering the highest viability percentages, in those that did not apply the pregerminative treatment. Viability tests showed that the most effective test, was tetrazolium test, because the embryo and the tissues can be observed directly. The Viability analysis contributed to know the possible influence of pregerminative treatments on the viability of the seeds. These are important factors since they are related to the germination of this tree with economic and forestry interest.

Keywords: Lixiviation, viability percentage, tetrazolium test, seed coat incision, Honduras. 


\section{INTRODUCCIÓN}

Guaiacum sanctum $L$. es una especie arbórea de crecimiento lento que pertenece a la familia Zygophyllaceae, habita en el bosque seco subtropical y tropical. En Centroamérica es conocida como guayacán, palo santo, guayacán real y lignum vitae en Norteamérica (Ferrufino-Acosta, Mejía-Ordóñez, \& Corrales-Andino, 2016). El guayacán es una especie de gran importancia en los bosques secos tropicales de Honduras, ya que es de especial interés económico debido a que su madera es una de las más densas y comúnmente se ha utilizado para la fabricación de propelas para barcos de vapor (Fuchs \& Hamrick, 2010). Además la corteza de esta especie produce una resina llamada guayacina (guayacol, o ácido alfa-gualacónico), la cual posee propiedades antibióticas y ha sido utilizada desde el año 1500 para el tratamiento de enfermedades de transmisión sexual, como la gonorrea y sífilis (Fuchs \& Hamrick, 2010). En la actualidad aún es utilizada como laxante y para el tratamiento de la tos, tuberculosis y reumatismo (Rivers, 2017).

Según, Rivers (2017) el habitat de G. sanctum se encuentra en disminución, debido principalmente a la deforestación asociada con el aumeno de las poblaciones humanas y la conversión de bosques a áreas de usos múltiples por el ser humano, por lo acual esta especie se encuentra dentro de la lista roja de especies amenazadas de la Unión Internacional para la Conservación de la Naturaleza (UICN) y también está catalogado en el apéndice II de la Convención sobre el Comercio Internacional de Especies Amenazadas de Fauna y Flora Silvestre (CITES).

Por otro lado la presión que existe en los recursos del ecosistema de los bosques secos, aumenta cada día, poniendo en peligro de extinción a varias especies nativas y endémicas. En la actualidad la restauración de los bosques secos, se traduce cada vez más en un fuerte compromiso político para proyectos de reforestación con árboles a gran escala. El valor de la utilización de especies nativas en la restauración del ecosistema, está recibiendo un creciente reconocimiento tanto entre los profesionales de la restauración, como entre los responsables políticos (Ferrufino, López, Suazo, \& Díaz, 2017).

El guayacán al ser una especie nativa amenazada de los bosques secos, ha recibido especial interés en cuanto a la realización de estudios para evaluar su proceso de germinación. Sin embargo la reproducción del $G$. sanctum es muy difícil en medio natural, no todas las semillas germinan fácilmente; por lo cual se requiere de un manejo especial que muchas veces incluye algún tratamiento pregerminativo, para 
romper la latencia de las semillas (Espinoza-Ocaña \& Orantes-García, 2014). Durante la latencia de la semilla, el crecimiento del embrión y las actividades metabólicas se reducen al mínimo cuando las condiciones no son favorables para la germinación (Sinha, 2014). La intensidad de la latencia se encuentra influenciada por varios factores ambientales, como ser la temperatura, la humedad y el ambiente gaseoso (Varela \& Arana, 2011). La germinación de las semillas está relacionada con la viabilidad de las mismas, la cual se define como, la capacidad que estas poseen para mantener vivo el embrión, para que logre germinar (Ríos de León, 2016). La viabilidad de las semillas, puede ser evaluada mediante diferentes pruebas, una de ellas es la prueba bioquímica de sales de tetrazolio (cloruro de 2, 3, 5, - trifenil-tetrazolio), el cual actúa en procesos de reducción-oxidación, formando una sustancia roja (trifenil-formazán) que colorea los tejidos vivos en el embrión (Victoria, Bonilla, \& Sánchez, 2006). Otra prueba utilizada para evaluar la viabilidad, es la flotabilidad la cual consiste en colocar las semillas en un recipiente con agua, indicando como viables a aquellas que se coloquen en el fondo del recipiente y como no viables aquellas que floten. Se considera que las semillas flotan y son no viables, debido a que el embrión ha muerto y se ha generado una cámara de aire, que permite la flotabilidad de la semilla (Varela \& Arana, 2011).

El objetivo de esta investigación fue analizar la viabilidad de las semillas de G. sanctum, posterior a la aplicación de dos tratamientos pregerminativos, a su vez se planteó como hipótesis, que la aplicación de tratamientos pregerminativos influyó en la viabilidad de las semillas de guayacán.

\section{MATERIALES Y MÉTODO}

\section{Área de estudio:}

El área de estudio estuvo comprendida en las cercanías del Estadio Carlos Miranda, del departamento de Comayagua (Figura 1) sitio donde se realizó la colecta de semillas de los individuos de guayacán o plantas madre, el mismo fue seleccionado con base en el estudio de Ferrufino-Acosta, Mejía-Ordóñez, \& Corrales-Andino, (2016) el cual señala que los promedios de mayor DAP (diámetro a la altura de pecho) fueron obtenidos en el departamento de Comayagua. Este factor fue considerado en el estudio ya que como lo menciona Ríos de León, (2016) la probabilidad de reproducción de G. sanctum aumenta con el tamaño del DAP, produciendo una mayor cantidad de frutos ( árboles $>35 \mathrm{~cm}$ DAP $>10,000$ frutos). 
Figura 1. Ubicación de individuos de guayacán muestreados

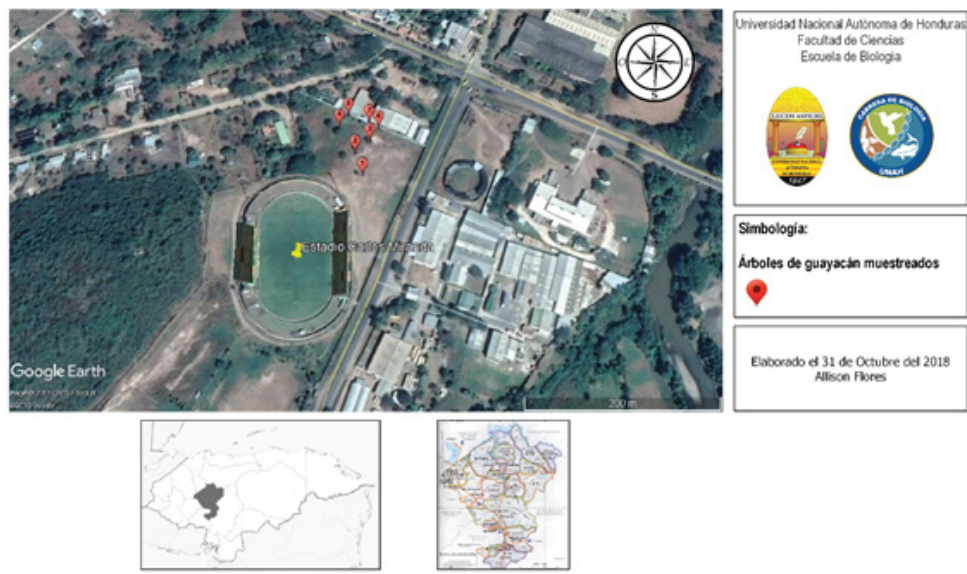

Elaboración: fuente propia

\section{Método de campo:}

La colecta de semillas fue realizada en Julio de 2018, muestreando un total de siete individuos de G. sanctum, a cada uno se le tomó el DAP, también se georreferenció cada árbol con un dispositivo de sistemas de geoposicionamiento global (GPS). La colecta fue realizada a mano alzada, extrayendo directamente los frutos de los árboles, o utilizando una pértiga en cada de que fuese necesario. Una vez colectados los frutos se extrajo las semillas de los mismos y se colocaron en sobres de papel manila y se refrigeraron a $4^{\circ} \mathrm{C}$ para evitar la formación de agentes patógenos. (Figura 2).

Figura 2. A. Individuo adulto de G. sanctum B. Fruto cápsula dehiscente maduro C. Semillas cubiertas por un arilo rojo carnoso

Elaboración: fuente propıa

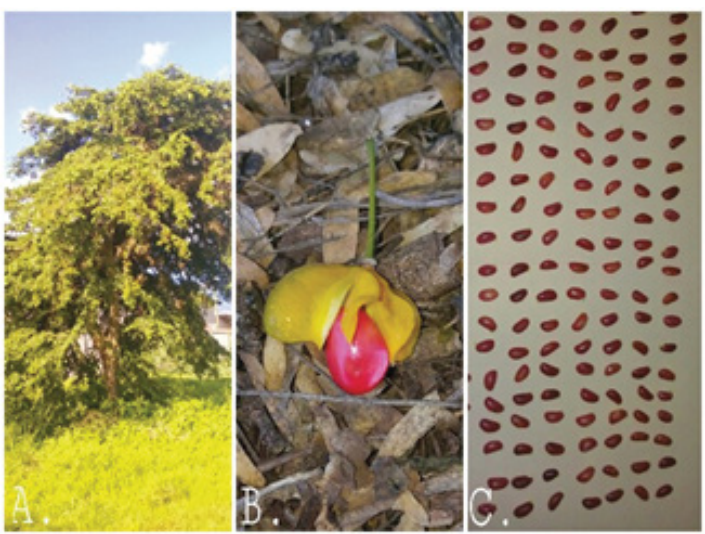




\section{Método de laboratorio:}

Una vez colectadas las semillas se dividieron en tres lotes, utilizando 120 semillas del total de la colecta. Uno de los lotes constituyó la prueba testigo y lo dos lotes restantes se les aplicó un tratamiento pregerminativo diferente.

1. Tratamiento pregerminativos: Cada tratamiento fue aplicado a diez semillas, y se realizaron tres réplicas de cada uno, conformando cada uno de los tres lotes con 40 semillas, dividiéndolos de la siguiente forma:

a) Prueba testigo: Sin aplicación de tratamiento pregerminativo.

b) Corte longitudinal en la testa: Se utilizó una navaja de afeitar y se realizó un corte en la testa en un extremo de la semilla.

c) Lixiviación: Para este tratamiento de sumergieron las semillas durante 24 horas en agua a temperatura ambiente.

Al concluir el proceso de aplicación de tratamientos pregerminativos, se sembraron las semillas en un semillero de 200 alveolos, utilizando fibra de coco como sustrato.

2. Pruebas de viabilidad: Transcurridos 45 días luego de la siembra, se extrajeron 36 semillas $(30 \%)$ del semillero y se sometieron a la pruebas de viabilidad 12 semillas $(10 \%)$ de cada lote. Las pruebas fueron realizadas en el laboratorio de Histología Vegetal y Etnobotánica Sonia Lagos-Witte, de la Escuela de Biología, de la Universidad Nacional Autónoma de Honduras.

a) Prueba de flotabilidad: Esta prueba consiste en colocar las semillas en un recipiente con agua, tomando como viables aquellas semillas que se depositen en el fondo del recipiente, y como no viables a aquellas que floten, una semilla que flota indica que es no viable, debido a que el embrión está muerto y ha generado una cámara de aire en la semilla que permite la flotabilidad de la misma (Varela \& Arana, 2011). Concluido el procedimiento de esta prueba se aplicó la segunda prueba y se calculó el porcentaje de viabilidad:

$\%$ Viabilidad= Número de semillas que no flotaron X 100

Número total de semillas sometidas a la prueba

b) Prueba de tetrazolio: Se realizó un corte transversal total en las semillas, con la ayuda de pinzas y navajas de afeitar, de forma que el embrión quedase expuesto totalmente. Con la ayuda de un gotero se humedeció papel filtro en su totalidad 
con la solución de Tetrazolio (TTC) al 1\%, y se colocó en las cajas petri, las semillas fueron colocadas con la ayuda de pinzas sobre el papel filtro, de forma que el embrión estuviese en contacto con el reactivo. Una vez rotuladas las cajas petri, fueron incubadas en un ambiente libre de luz y humedad durante 24 horas, esto para evitar la interferencia de luz en las reacciones de oxidación-reducción (Figura 3). De igual forma se calculó el porcentaje de viabilidad para esta prueba:

$\frac{\% \text { Viabilidad }=\text { Número de semillas teñidas } \times 100}{\text { Número total de semillas sometidas a la prueba }}$

Figura 3. Procedimiento realizado para la prueba de tetrazolio

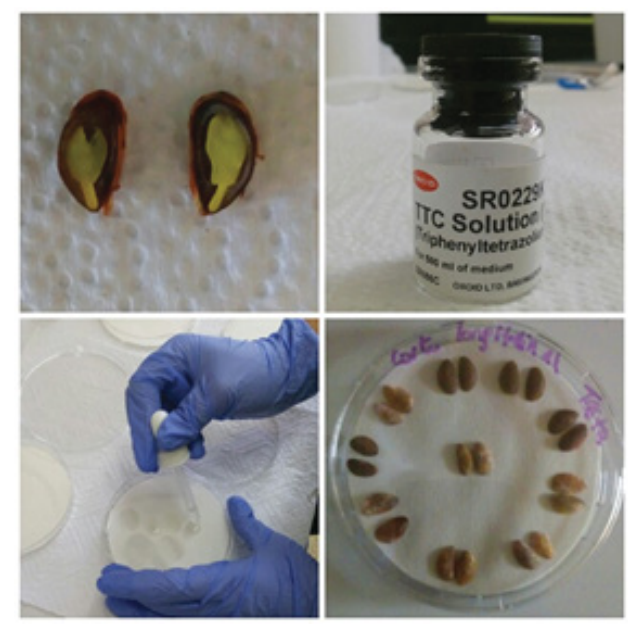

Elaboración: fuente propia

\section{RESULTADOS}

De acuerdo con el DAP tomado a los individuos el promedio fue de 32.65 unidades, lo que concuerda con los resultados obtenidos en el estudio realizado por Ferrufino-Acosta, Mejía-Ordóñez, \& Corrales-Andino, (2016), en el cual se obtuvo un promedio de DAP entre 30-35 unidades, en las poblaciones de G. sanctum de Comyagua (Figura 4).

Al concluir los tratamientos pregerminativos y las pruebas de viabilidad se calcularon los porcentajes de viabilidad de las pruebas aplicadas a cada uno de los lotes de semillas. (Cuadro 1 y 2 ). 
Figura 4. DAP de individuos muestreados.

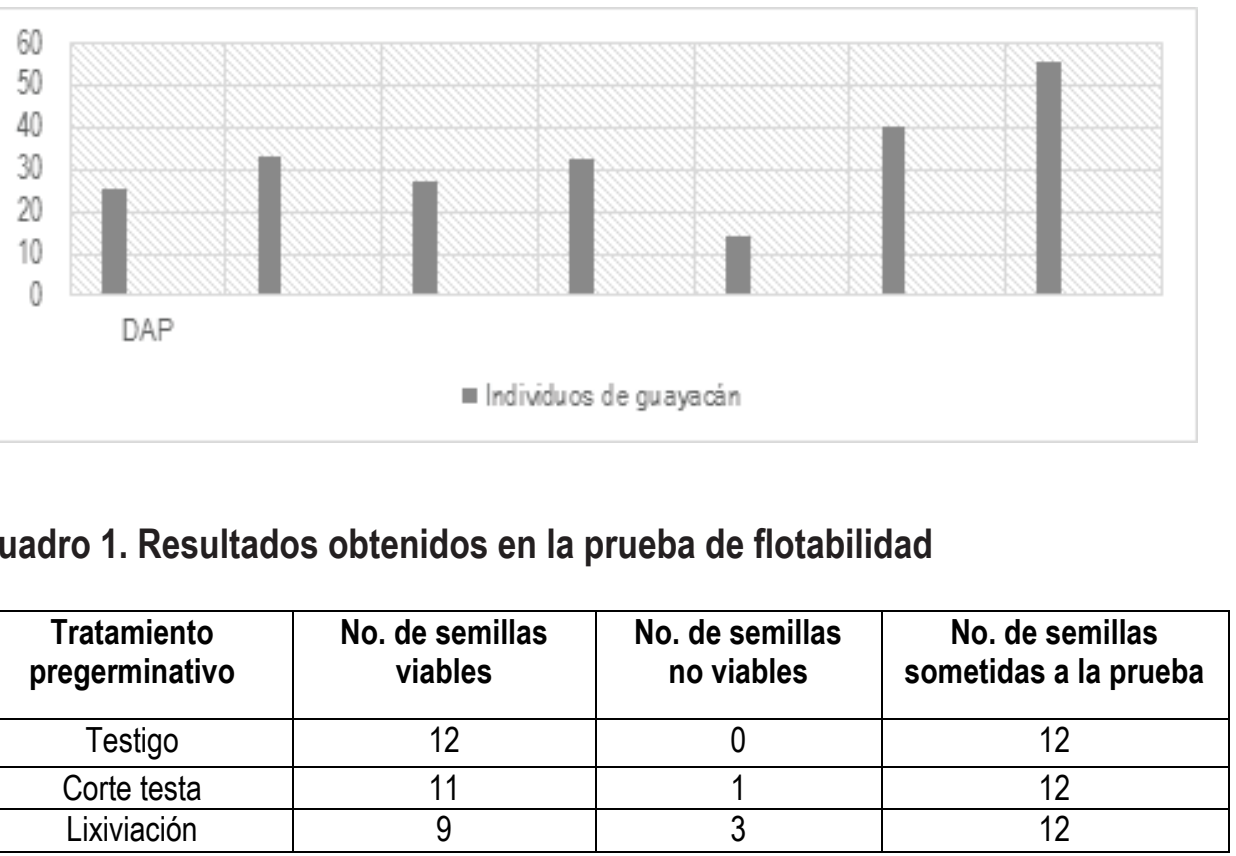

Cuadro 2. Resultados obtenidos en la prueba de tetrazolio

\begin{tabular}{|c|c|c|c|}
\hline $\begin{array}{c}\text { Tratamiento } \\
\text { pregerminativo }\end{array}$ & $\begin{array}{c}\text { No. de semillas } \\
\text { viables }\end{array}$ & $\begin{array}{c}\text { No. de semillas no } \\
\text { viables }\end{array}$ & $\begin{array}{c}\text { No. de semillas sometidas } \\
\text { a la prueba }\end{array}$ \\
\hline Testigo & 11 & 1 & 12 \\
\hline Corte testa & 9 & 3 & 12 \\
\hline Lixiviación & 5 & 7 & 12 \\
\hline
\end{tabular}

Finalizadas las pruebas, se tomó únicamente las semillas viables (Cuadro 3) para calcular los porcentajes de viabilidad (Figura 4).

Cuadro 3. Se muestran los totales de semillas viables obtenidos para cada tratamiento pregerminativo utilizado.

\begin{tabular}{|l|c|c|c|}
\hline $\begin{array}{c}\text { Tratamiento } \\
\text { pregerminativo }\end{array}$ & Flotabilidad & Tetrazolio & TOTAL \\
\hline Testigo & 12 & 11 & $\mathbf{2 3}$ \\
\hline Corte en testa & 11 & 9 & $\mathbf{2 0}$ \\
\hline Lixiviación & 9 & 5 & $\mathbf{1 4}$ \\
\hline
\end{tabular}


Figura 4. Se muestran las comparaciones de los porcentajes obtenidos en las pruebas de viabilidad

Porcentajes de viabilidad

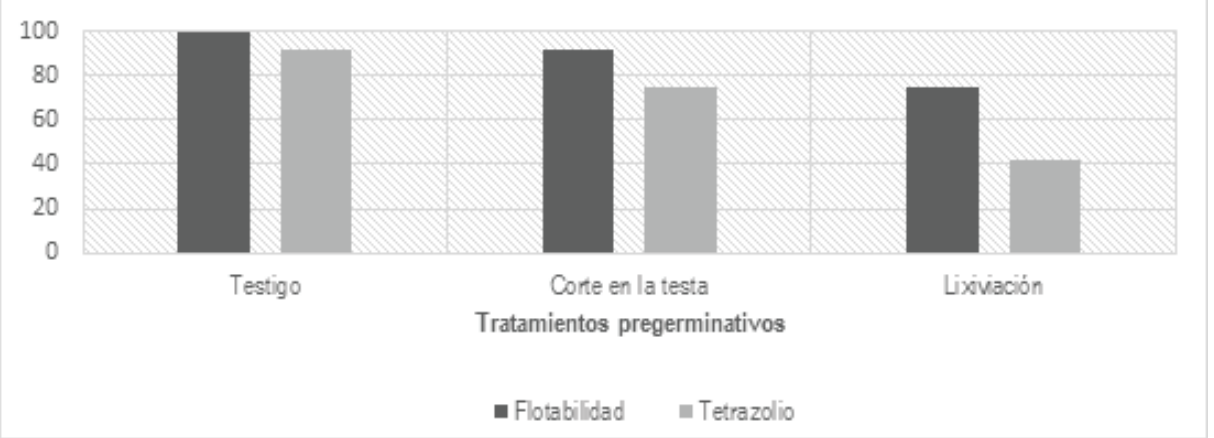

\section{DISCUSIÓN}

La aplicación de tratamientos pregerminativos puede variar de acuerdo a la morfología de la semilla, de las especies vegetales Taylor, (1966) señala que la latencia fisica de G. sanctum es provocada por el arilo y la testa de la semilla, debido a que contiene compuestos que inhiben la germinación, una vez que estas estructuras son removidas, las semillas logran germinar, sin embargo bajo condiciones naturales, en particular en la época lluviosa las semillas germinan rápidamente.

Los tratamientos pregerminativos aplicados en este estudio consistieron en lixiviación, tratamiento mediante el cual las semillas son remojadas en agua corriente con la finalidad de remover los inhibidores químicos presentes en la testa. Este tratamiento también es empleado con el objetivo de ablandar la testa (Varela \& Arana, 2011). El segundo tratamiento pregerminativo aplicado fue el corte en la testa, este permite la entrada de agua y acelera el proceso de germinación (Sánchez, 1995). Como ya se mencionó, los tratamientos pregerminativos estan enfocados en acelerar el proceso de germinación sin embargo en los dos meses de duración de este estudio, no se registró germinación en las semillas, es por ello que se procedió a analizar la viabilidad de las mismas.

Con base en los resultados obtenidos en la prueba de viabilidad de flotabiliad, el mayor porcentaje registrado fue en el lote de semillas testigo con un $100 \%$, seguido del lote de corte en testa con $91 \%$, y finalmente el lote de lixiviación con $75 \%$. Comparando con los resultados obtenidos en la prueba de viabilidad de tetrazolio, existió 
una notable diferencia en los porcentajes, ya que el lote testigo presentó un $91 \%$, el corte en testa $75 \%$ y el lote de lixiviación registró el porcentaje más bajo con un $41 \%$. Realizando una comparación de estos porcentajes, se logra determinar que las semillas a las cuales no se les aplicó ningún tratamiento presentaron una mayor viabilidad, siendo esto un posible indicativo que la aplicación de tratamientos pregerminativos, no es totalmente necesaria para acelerar el proceso de germinación en esta especie vegetal.

El corte en testa registro el siguiente mayor numero de semillas viables, para esta especie indicando que este tratamiento es más optimo que la lixiviación, tratamiento que registró el menor número de semillas viables, contrario a lo descrito por, Espinoza-Ocaña \& Orantes-García, (2014) quienes establecen en su estudio de viabilidad de semillas de $G$. sanctum, que el mayor porcentaje de semillas viables que lograron germinar fueron aquellas que se sometieron al tratamiento de lixiviación.

En cuanto a las pruebas de viabilidad realizadas, es importante resaltar la confiabilidad de las mismas ya que ambas pruebas fueron aplicadas a las mismas semillas, comenzando con la prueba de flotabilidad y posteriormente con la prueba de tetrazolio. Se observó que algunas semillas que fueron clasificadas como viables en la prueba de flotabilidad, al ser sometidas a la prueba de tetrazolio no presentaron tinción en el embrión, es decir fueron semillas no viables (Figura 5).

\section{Figura 5. A. Semilla clasificada como viable según la prueba de flotabilidad pero como no viable según la prueba de tetrazolio. B. Semilla no viable para ambas pruebas.}

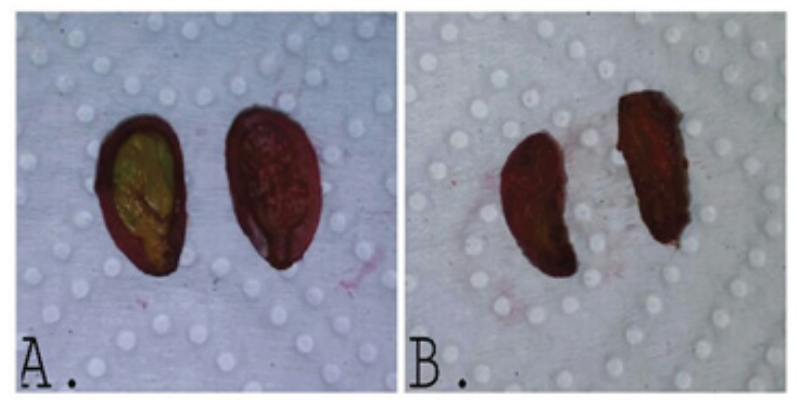

En la figura 5A se observa el embrión. Al aplicar la prueba de flotabilidad a este grupo se determinó que la viabilidad fue positiva; sin embargo, cuando estas mismas semillas se sometireron a la prueba de tetrazolio, el resultado fue de viabilidad negativa, ya que no hubo tinción lo cual indica muerte del tejido embrional. Esto a su vez indica que la prueba de flotabilidad puede dar como resultado un falso positivo y no es 
suficiente por si sola para determinar la viabilidad del embrión. En la la figura $5 B$, se observa una semilla no viable para ambas pruebas, ya que el embrión se encuentra ausente.

En la prueba de tetrazolio (TTC) se utilizó el reactivo al 1\%, sin embargo la concentración de este puede variar de acuerdo a la especie vegetal, en estudio previos de viabilidad de semillas de $\mathrm{G}$. sanctum, se utilizó una concentración al 0,5\% (Espinoza-Ocaña \& Orantes-García, 2014). También se han realizado estudios de viabiliad en G. sanctum utilizando TTC al 1\% (Ríos de León, 2016). Sin embargo Hernández, Lobo, Medina, Régulo-Cartagena, \& Delgado, (2009) mencionan que se obtiene una mejor tinción con una concentración al 1\% de TTC.

Otro factor importante en la prueba de viabilidad de tetrazolio, es la tinción de los embriones con la sustancia trifenil-formazan. Las semillas viables son coloreadas completamente y aquellas con ausencia de coloración son no viables, en algunos casos estas pueden colorearse parcialmente. Se han establecido diferentes grados de tinción en regiones esenciales (radícula, plúmula, eje embrional y cotiledones) (Figura 6) (Victoria, Bonilla, \& Sánchez, 2006).

\section{Figura 6. A y B. Semillas parcialmente teñidas. C. Semilla con tinción total.}

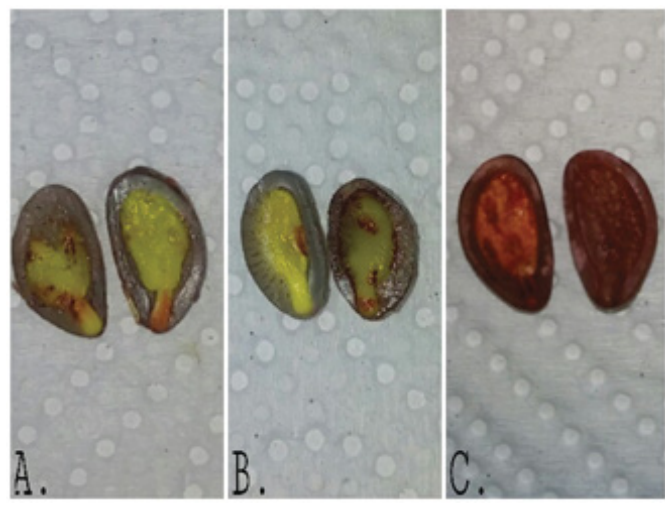

En la figura $6 \mathrm{~A}$ y $6 \mathrm{~B}$, se muestran semillas con una tinción parcial en los embriones, pero tal como lo mencionan, Victoria, Bonilla, \& Sánchez, (2006) se observa tinción en zonas esenciales del embrión, en este caso la radícula y la plúmula. Cabe mencionar que las semillas que se observan en la Figura $6 \mathrm{~A}$ y $6 \mathrm{~B}$, fueron sometidas a la prueba de viabilidad de tetrazolio durante 24 horas, y al observarse la tinción parcial se decidió someterlas durante 24 horas más, para observar posibles cambios que en este caso se manifestaron provocando una tinción total en el embrión, tal como se observa en la Figura 6C. 


\section{CONCLUSIONES}

El análisis de viabilidad de semillas de guayacán, posterior a la aplicación de dos tratamientos pregerminativos, contribuyó a conocer la posible influencia de dichos tratamientos en la viabilidad de las semillas. Estos son factores importantes ya que se encuentran relacionados con la germinación de esta especie vegetal de especial interés económico y forestal. Con base en los resultados se demostró que aquellas semillas a las cuales no se les aplicó un tratamiento pregerminativo, el lote testigo en este caso, presentaron un mayor número de semillas viables, aptas para germinar. En las pruebas de viabilidad existió una notable variación en la efectividad de las mismas, siendo más efectiva la prueba de tetrazolio, ya que esta prueba permite observar de forma directa el embrión y el estado de sus tejidos embrionarios.

Con base en los resultados obtenidos, no se recomiendan estos tratamientos pregerminativos para esta especie; sin embargo, hay que tener en cuenta que otros factores ajenos a los tratamientos, pudieron verse involucrados en la muerte del embrión y a su vez en la viabilidad de las semillas, esto puede estar relacionado con factores abióticos, como humedad, sustrato, luz, y temperatura, estos no fueron medidos en este estudio, pero es recomendable realizar estudios posteriores tomando en cuenta estos factores y la aplicación de nuevas pruebas de viabilidad y nuevos tratamientos pregerminativos para realizar las respectivas comparaciones.

\section{AGRADECIMIENTOS}

La autora expresa su especial agradecimiento, a las asesoras de esta investigación, Lilian Ferrufino Ph.D y Virna López Ph.D. por sus aportes, comentarios y observaciones. Al laboratorio de Histología Vegetal y Etnobotánica Sonia Lagos-Witte, de la Escuela de Biología y al Laboratorio Teasdale-Corti de la Escuela de Microbiología, por la facilitación del reactivo Trifeniltetrazolio. También se agradece la colaboración de las estudiantes de la carrera de Biología, Shasling Pacheco y Ana Reyes en el trabajo de campo y al estudiante de la carrera de Microbiología, Isaac Sabillón por su apoyo en el trabajo de laboratorio. 


\section{REFERENCIAS BIBLIOGRÁFICAS}

Espinoza-Ocaña, L., \& Orantes-García, C. (Junio de 2014). Viabilidad y germinación de Guaiacum sanctum L. (Zygophyllaceae), árbol tropical amenazado. LACANDONIA, 8(1), $37-40$.

Ferrufino, L., López, T., Suazo, L., \& Díaz, R. (25 de Mayo de 2017). La colección de especies nativas del bosque seco tropical de Honduras: un laboratorio de campo. Centro de Investigación Científica de Yucatán, 93-97.

Ferrufino-Acosta, L., Mejía-Ordóñez, T. M., \& Corrales-Andino, R. E. (Diciembre de 2016). Estudio Poblacional de Guaiacum sanctum L. (Zygophyllaceae) en los bosques secos de Honduras. Revista Ciencia y Tecnología(19), 78-93.

Fuchs, E., \& Hamrick, J. (2010). Genetic Diversity in the Endagered Tropical Tree Guaiacum sanctum (Zygophyllaceae). Journal of Heredity, 101(3), 284-291.

Hernández, M. I., Lobo, M., Medina, C. I., Régulo-Cartagena, J., \& Delgado, O. A. (2009). Comportamiento de la germinación y categorización de la latencia en semillas de mortiño (Vaccinium meridionale Swartz). Agronomía Colombiana, 27, 15-23.

Ríos de León, N. d. (Noviembre de 2016). Germinación in vitro de Guaiacum sanctum L. (Zygophyllaceae) como alternativa de conservación y aprovechamiento sustentable. Universidad de Ciencias y Artes de Chiapas. Instituto de Ciencias Biológicas , 1-95.

Rivers, M. (2017). Guaiacum sanctum. The IUCN Red List of Threatened Species 2017. Obtenido de http://dx.doi.org/10.2305/IUCN.UK.2017-3.RLTS.T32955A68085952.en

Sánchez, D. (Octubre de 1995). Determinación del potencial germinativo de tres especies maderables nativas de un bosque húmedo tropical de Nicaragua. Avances en la producción de semillas forestales en Ámerica Latina, 203-206.

Sinha. (2014). Modern Plant Physiology. Oxford: Alpha Science Internantional.

Taylor, A. (1966). Seed germination and seedling growth of Guaiacum sanctum L. Florida State Horticultural Society, 79, 468-470.

Varela, S., \& Arana, V. (2011). Latencia y Germinanción de Semillas. Tratamientos Pregerminativos. Serie ténica: Sistemas Forestales Integrados, 1-10.

Victoria, J., Bonilla, C., \& Sánchez, M. (2006). Viabilidad en tetrazolio de semillas de caléndula y eneldo. Acta Agronómica, 55(1), 1-15. 